\title{
Widespread high grade prostatic intraepithelial neoplasia on biopsy predicts the risk of prostate cancer: A 12 months analysis after three consecutive prostate biopsies
}

\author{
Cosimo De Nunzio ${ }^{1}$, Simone Albisinni ${ }^{1}$, Antonio Cicione ${ }^{1}$, Mauro Gacci ${ }^{2}$, \\ Costantino Leonardo ${ }^{1}$, Francesco Esperto ${ }^{1}$, Andrea Tubaro ${ }^{1}$ \\ ${ }^{1}$ Department of Urology, Ospedale Sant'Andrea, University "La Sapienza", Rome, Italy; \\ ${ }^{2}$ Department of Urology, Ospedale Careggi, University of Florence, Italy.
}

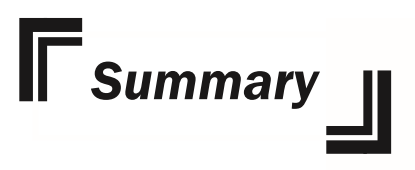

Purpose: To evaluate the risk of prostate cancer (PCa) on a third prostate biopsy in a group of patients with two consecutive diagnoses of high grade intraepithelial neoplasia (HGPIN).

Materials and methods: From November 2004 to December 2007, patients referred to our clinic with a PSA $\geq 4 \mathrm{ng} / \mathrm{ml}$ or an abnormal digital rectal examination (DRE) were scheduled for trans-rectal ultrasound (TRUS) guided 12-core prostate biopsy. Patients with HGPIN underwent a second prostate biopsy, and if the results of such procedure yielded a second diagnosis of HGPIN, we proposed a third 12-core needle biopsy regardless of PSA value. Crude and adjusted logistic regressions were used to assess predictors of PCa on the third biopsy.

Results: A total of 650 patients underwent 12 cores transrectal ultrasound prostatic biopsy in the study period. Of 147 (22\%) men with a diagnosis of HGPIN, 117 underwent a second prostatic biopsy after six months and 43 a third biopsy after other six months. After the third biopsy, 19 patients (34\%) still showed HGPIN, 15 (35\%) were diagnosed with PCa and 9 (21\%) presented with chronic prostatitis. Widespread HGPIN on a second biopsy was significantly associated with PCa on further biopsy $\left(\chi^{2}=4.04, p=0.04\right)$. Moreover, the presence of widespread HGPIN significantly predicted the risk of PCa on crude and adjusted logistic regressions.

Conclusions: Widespread HGPIN on second biopsy is associated with the presence of PCa on a third biopsy. Nonetheless, the relationship between HGPIN and PCa remains complex and further studies are needed to confirm our findings.

KEY WORDS: Prostate cancer; High grade prostatic intraepithelial neoplasia; Biopsy; Gleason score; Widespread.

\section{INTRODUCTION}

High grade prostatic intraepithelial neoplasia (HGPIN) is a cytoarchitectural modification of the prostatic tissue, with pre-existing acini and ducts lined by cytologically atypical cells (1). It has long been considered the pre-neoplastic lesion of prostate cancer (PCa) $(1,2)$ and is considered a risk factor for PCa on subsequent biopsy (3-7). The prognostic value of HGPIN in prostate biopsy cores however has been questioned and controversy has arisen on whether patients with a diagnosis of HGPIN should undergo further biopsies $(2,8,9)$. Widespread HGPIN, defined as $\geq 4$ biopsy cores involved with the intraepithelial lesion, has been found to be significantly associated with PCa diagnosis on further biopsy by different investigators $(6,10-15)$, including our group (16). Other predictors of PCa on a subsequent biopsy in patients with isolated HGPIN, such as age, an abnormal digital rectal examination (DRE), an abnormal prostate volume, PSA, PSA ratio or PSA density values have been examined, yet no 
consensus on their predictive role has been reached $(5,7$, $14,17)$. To date, the prognostic value of HGPIN, clinical markers (age, digital rectal examination, PSA, etc) and widespread HGPIN in men after multiple diagnoses of isolated HGPIN remains controversial, and little is available on long term follow-up of these patients.

Data confirming a positive association of widespread HGPIN and PCa diagnosis on repeat biopsy have already been published by our group (16). We now report the results after the third biopsy in men with two consecutive diagnoses of isolated HGPIN. We explored the association of HGPIN, widespread HGPIN and clinical markers (age, digital rectal examination, PSA, etc) and PCa risk on a third biopsy, in order to elucidate the potential predictive role of HGPIN on PCa and further help to indentify the correct clinical management for patients with HGPIN.

\section{MATERIALS AND METHODS}

From November 2004 to December 2007, after receiving institutional review board approval, patients referred to our clinic with a PSA $\geq 4 \mathrm{ng} / \mathrm{ml}$ or an abnormal digital rectal examination (DRE) were scheduled for trans-rectal ultrasound (TRUS) guided 12-core prostate biopsy after informed consent was signed. In every patient diagnosed with HGPIN, a second biopsy was proposed after 6 months regardless of PSA values. Finally, in patients with a second diagnosis of HGPIN a third and final biopsy was proposed 6 months after the second procedure, for a total of 12 months follow-up.

Biopsy was performed as an outpatient procedure and the methodology has been throughout fully described in previously published peer-reviewed manuscripts $(16,18)$. All biopsies were performed following the same 12-core scheme. Before each procedure, blood specimens were obtained and free and total PSA were measured. Prostate volume was calculated by TRUS. Patients on finasteride or dutasteride and men who had undergone prostate surgery were excluded from the study.

A single uro-pathologist performed the histological evaluation for all biopsy series. The histological/architectural threshold used to assign the various diagnoses was that proposed by the WHO $(19,20)$. In areas suspicious for ASAP or HGPIN, immunohistochemical staining of sequential sections was used to confirm the eventual loss of basal cells using a mix of anti-p63 and $34 \beta 12$ cytokeratin antibodies. As defined by Netto and Epstein, widespread HGPIN was defined as 4 or more cores involved with HGPIN (21).

\section{Statistical analysis}

Widespread HGPIN on the second biopsy was examined as a categorical variable. The presence or absence of cancer on the third biopsy specimens defined our main categorical outcome variable. We performed chi-square test to evaluate the association between widespread HGPIN on the second biopsy and the diagnosis of $\mathrm{PCa}$ on the subsequent biopsy. Crude and adjusted logistic regressions were used to evaluate the association of clinical and pathological predictors and the risk of PCa on the third biopsy. However, given the small number of events in our model, we executed separate multivariate analyses for each predictor other than widespread HGPIN: multivariate analyses constantly included the presence of widespread HGPIN on the second biopsy (categorical) plus a second term as age, PSA, TRUS volume, DRE, PSA ratio and PSA Density. Due to non-parametrical distribution, PSA values and derivates (PSA ratio and density) were logarithmically transformed in the multivariate logistic regression tests. Mann-Whitney test was used to explore differences in age, prostate volume, PSA concentration, PSA ratio and PSA density across our two outcome groups and between men with and without widespread HGPIN at second biopsy. Wilcoxon signed rank sum test was used to evaluate significant modifications of PSA concentration, ratio and density between the second and third biopsy. Statistical analysis was performed using STATA 11 (StataCorp, College Station, TX).

\section{RESULTS}

During the study period 650 men underwent primary prostate biopsy. Of these, $147(22 \%)$ were diagnosed with HGPIN. As 30 men refused further procedures, a second biopsy was performed in 117 men, six months later. Data regarding the second biopsy have already been published (16). Out of 117 re-biopsies, 75 (64\%) yielded a second diagnosis of HGPIN and to these men a third prostate biopsy was proposed, 6 months after the second biopsy. 22 of these patients refused to undergo the third biopsy and 10 underwent prostate surgery for bladder outlet obstruction; no cancer was found in any of the pathological specimens examined after surgery in these 10 patients. 43 men were therefore available for final analysis. Patients characteristic are illustrated in Table 1. After the third biopsy, 19 patients (44\%) still showed HGPIN, 15 (35\%) were diagnosed with PCa and $9(21 \%)$ presented with chronic prostatitis. A flow chart (figure 1) clearly illustrates the results of the biopsies. The 10 men who underwent prostate resection for bladder outlet obstruction were all diagnosed with benign prostatic hypertrophy.

Table 1.

Clinical characteristics of the cohort (43 patients).

\begin{tabular}{|l|c|}
\hline Age $(\mathrm{yrs})$ & Median (IQR) \\
\hline Prostate volume $(\mathrm{ml})$ & $65(61-70)$ \\
\hline PSA $(\mathrm{ng} / \mathrm{ml})$ & $56(42-64)$ \\
\hline PSA ratio $(\%)$ & $7.53(5.87-10.8)$ \\
\hline PSA Density $\left(\mathrm{ng} / \mathrm{ml}^{2}\right)$ & $15(12-22)$ \\
\hline DRE Negative & $0.14(0.10-0.22)$ \\
\hline Positive & $37(86 \%)$ \\
\hline $\begin{array}{l}\text { Widespread HGPIN at second biopsy } \\
(\geq 4 \text { cores) }\end{array}$ & $6(14 \%)$ \\
\hline
\end{tabular}


Figure 1.

Study design.

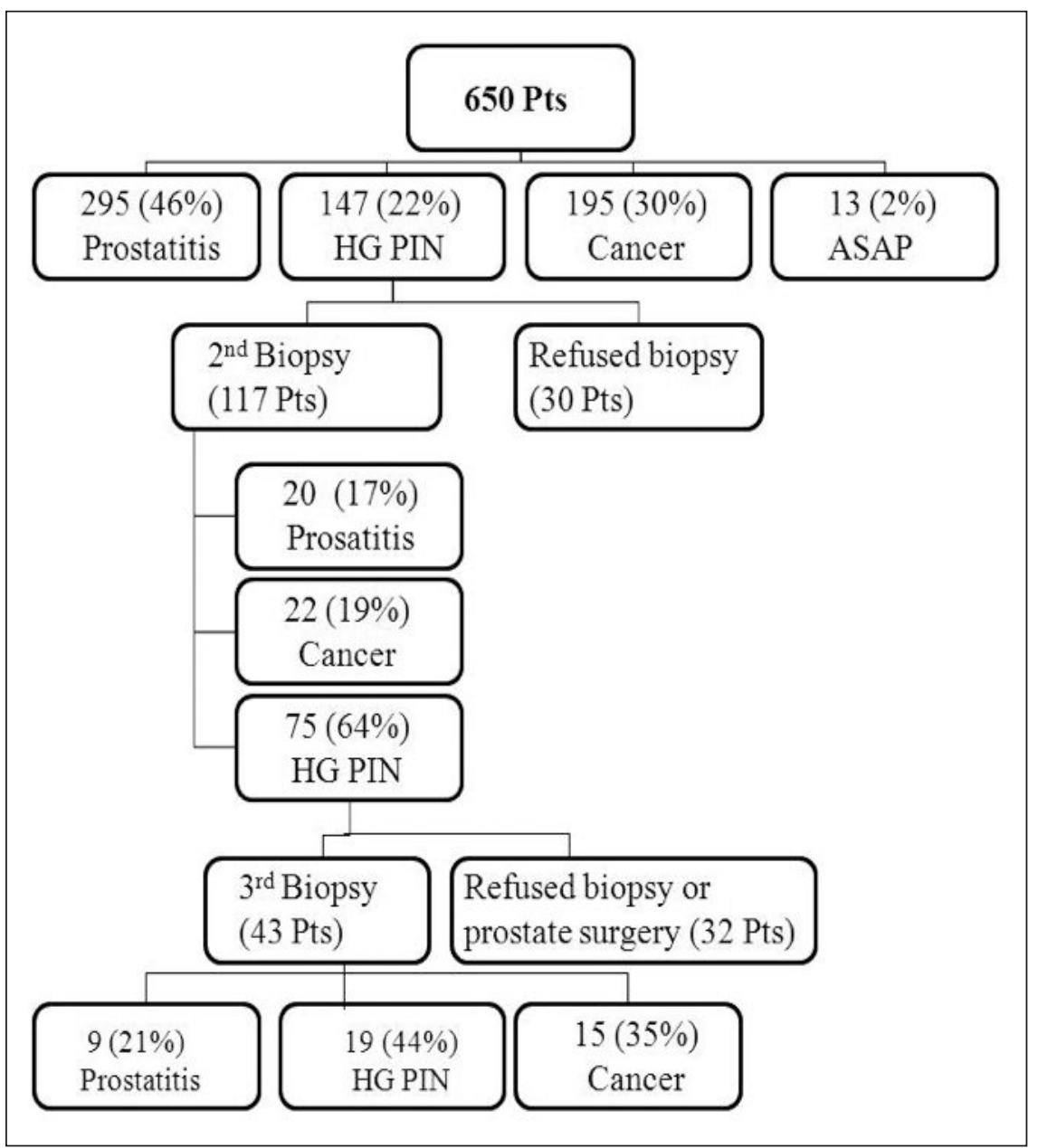

Of the 15 patients with $\mathrm{PCa}, 9$ had a low grade Gleason $6(3+3)$ adenocarcinoma, 4 men had a Gleason $7(3+4)$ tumor, while only one Gleason $8(4+4)$ and one Gleason $9(4+5)$ cancers were diagnosed. A single core was involved in 10 of the men with cancer, with a $15 \%$ median core cancer extension. Of these, 7 were Gleason $6(3+3)$ and the remaining 3 were Gleason 7 $(3+4)$. Two cores were positive for cancer in 4 patients with a median extension of $15 \%$. In one patient, diagnosed with a Gleason $8(4+4), 4$ cores were involved with cancer, for a maximum of $60 \%$ of their length.

No significant difference in the distribution of age, PSA, prostate volume, DRE, PSA ratio and PSA density (at the time of third biopsy) was found across the two outcome groups (Table 2).

Widespread HGPIN on a second biopsy was significantly associated with PCa on further biopsy $(\chi 2=4.04, p=0.04)($ Table 2$)$. Moreover, the presence of widespread HGPIN significantly predicted the risk of $\mathrm{PCa}$ on crude logistic regression (OR 3.75, 95\% CI 1.00-14.02, $\mathrm{p}=0.049$ ). Widespread HGPIN remained a significant predictor of $\mathrm{PCa}$ on all

Table 2.

Clinical and pathological differences across groups.

\begin{tabular}{|c|c|c|c|c|c|c|}
\hline & No Cancer & Cancer & p-value ${ }^{1}$ & $<4$ cores involved & $\begin{array}{l}\geq 4 \text { cores involved } \\
\text { (Widespread HGPIN) }\end{array}$ & $p$-value ${ }^{1}$ \\
\hline Number of patients & $28(65 \%)$ & $15(35 \%)$ & - & $26(60 \%)$ & $17(40 \%)$ & - \\
\hline $\begin{array}{l}\text { Age (yrs) } \\
\text { Median (IQR) }\end{array}$ & $66(60-70)$ & 65 (61-71) & 0.86 & $66(62-71)$ & $64(57-70)$ & 0.30 \\
\hline $\begin{array}{l}\text { Prostate volume (ml) } \\
\text { Median (IQR) }\end{array}$ & $58(43-65)$ & $51(38-64)$ & 0.31 & $57(40-65)$ & $51(45-63)$ & 0.80 \\
\hline $\begin{array}{l}\text { DRE negative } \\
\text { positive }\end{array}$ & $\begin{array}{c}25(89 \%) \\
3(11 \%)\end{array}$ & $\begin{array}{c}12(80 \%) \\
3(20 \%)\end{array}$ & 0.402 & $\begin{array}{c}22(85 \%) \\
4(15 \%)\end{array}$ & $\begin{array}{c}15(88 \%) \\
2(12 \%)\end{array}$ & $0.74^{2}$ \\
\hline $\begin{array}{l}\text { PSA (ng/ml) } \\
\text { Median (IQR) } \\
\end{array}$ & $6.86(5.66-9.3)$ & $8.84(6.75-13.5)$ & 0.14 & $7.8(5.88-11.7)$ & $6.86(5.87-8.89)$ & 0.39 \\
\hline $\begin{array}{l}\text { PSA ratio (\%) } \\
\text { Median (IQR) }\end{array}$ & $16(12-24)$ & $15(10-19)$ & 0.27 & $16(12-25)$ & $15(12-20)$ & 0.72 \\
\hline $\begin{array}{l}\text { PSA Density }\left(\mathrm{ng} / \mathrm{ml}^{2} \text { ) }\right. \\
\text { Median (IQR) }\end{array}$ & $0.13(0.10-0.21)$ & $0.15(0.1-0.26)$ & 0.24 & $0.145(0.10-0.23)$ & $0.14(0.10-0.18)$ & 0.57 \\
\hline Widespread HGPIN & $8 / 28(29 \%)$ & $9 / 15(60 \%)$ & 0.042 & - & - & - \\
\hline Prostate cancer & - & - & - & $6 / 26(23 \%)$ & 9/17 (53\%) & 0.042 \\
\hline
\end{tabular}

${ }^{1}$ Mann-Whitney test.

${ }^{2} \chi^{2}$ test. 
Table 3.

Multivariate logistic regressions: exploring the risk of prostate cancer on third biopsy.

\begin{tabular}{|lccc}
\hline & OR & $\mathbf{9 5 \%}$ CI & p-value \\
\hline Widespread HGPIN $^{\prime}$ & 3.75 & $1.00-14.02$ & 0.04 \\
\hline Age $^{1}$ & 1.04 & $0.93-1.15$ & 0.51 \\
\hline PSA $^{1,2}$ & 3.98 & $0.83-19.02$ & 0.08 \\
\hline Prostate volume $^{1}$ & 1.00 & $0.97-1.04$ & 0.81 \\
\hline DRE $^{1}$ & 2.53 & $0.39-16.19$ & 0.98 \\
\hline PSA ratio $^{1,2}$ & 0.37 & $0.10-1.35$ & 0.13 \\
\hline PSA Density $^{1,2}$ & 2.89 & $0.84-9.94$ & 0.09 \\
\hline
\end{tabular}

1 Due to the small number of events separate regressions were performed, adding each single term to the initial model with our main predictor variable (widespread HGPIN) (see text).

2 PSA, PSA ratio and PSA density were log-transformed due to non-parametrical distribution.

multivariate models (all $\mathrm{p}<0.05$ ). All clinical parameters evaluated, such as age, PSA, DRE, prostate volume and PSA ratio were not significant predictors of cancer at the time of the third prostate biopsy (Table 3). No significant differences in age, prostate volume, DRE, PSA, PSA ratio and PSA density were found between men with and without widespread HGPIN (Table 2). PSA concentration was not significantly modified between the second and third biopsy (median [IQR]: 7.83 [5.34$10.50]$ vs. 7.53 [5.87-10.80], $\mathrm{p}=0.34)$. The presence or absence of widespread HGPIN on the second biopsy did not significantly differ across patients with chronic prostatitis and patients with HGPIN on third biopsy ( $\mathrm{p}=0.12)$.

Finally, of the 43 patients who underwent the full set of three biopsies, 12 had a diagnosis of widespread HGPIN at the time of the first biopsy. Of these, 9 (75\%) were rediagnosed with widespread HGPIN on the second biopsy, while the remaining 3 (25\%) had focal HGPIN at that time. Cancer was found on third biopsy only in the first 9 patients (those with widespread lesions on both biopsies, in particular in 5 of these 9 men (56\%), while none of the 3 patients with widespread HGPIN only on the first biopsy had a diagnosis of PCa on the third biopsy.

\section{Discussion}

HGPIN is a common pathological finding on prostate biopsy and has been associated with an increased risk of PCa on subsequent biopsies $(3-6,9)$. Initially this risk was estimated around 50\% (4), however studies performed after 2000, in the era of extended prostate biopsy, have shown that this risk is approximately $23 \%$, compared to a $19 \%$ risk of detecting cancer after a benign diagnosis (2). The impact of HGPIN on the need for further biopsies has thus been redimensioned, and numerous studies have explored pathological features of HGPIN in order to predict PCa on subsequent biopsies $(2,21)$. In this context, the denomination of widespread or multifocal HGPIN has arisen, defined by Netto and
Epstein as $\geq 4$ cores involved with HGPIN (21). This pathological entity has been positively associated with a significantly increased risk of PCa in numerous studies $(6,7,10-14,16)$, ranging from $36 \%$ to $39 \%$. To date only few studies $(12,14,22-25)$ have explored the risk of cancer following multiple biopsies ( $>2$ procedures) diagnosing HGPIN; moreover only two manuscripts have examined the cancer risk at third biopsy after diagnosing multiple cores involved with HGPIN on a second prostate biopsy $(12,14)$. In this manuscript we addressed this issue by conducting a prospective trial with a minimum 12 month follow-up, during which men with two consecutive diagnoses of HGPIN underwent a third prostate biopsy. Widespread HGPIN on the second biopsy was significantly associated with the risk of PCa. No clinical parameter such as age, DRE, prostate volume, PSA, PSA Density or PSA ratio was able to significantly predict cancer. If validated, these results strengthen the prognostic value of widespread HGPIN, with impact on the need for further oncologic surveillance in patients with such diagnosis

We found a significant association between widespread HGPIN on second biopsy and PCa $\left(\chi^{2}=4.04, \mathrm{p}=0.04\right)$, and men with widespread HGPIN had a 4-fold, significant increase in risk of detecting $\mathrm{PCa}$ on subsequent biopsy compared to men with 3 or less cores involved with HGPIN. The overall cancer risk on the third biopsy for men with widespread HGPIN on second prostate biopsy was 53\%, higher that the risk if widespread was present at the time of the first biopsy (36-39\% risk). In line with these findings are the results reported by Bishara et al, who found a 50\% cancer risk if multiple cores $(\geq 2)$ involved with HGPIN had been found on second biopsy (12). Abdel-Khaled et al. reported a similar $58 \%$ risk in patients with multifocal HGPIN (14). Whether these results justify the need to perform an early re-biopsy (6 months) in patients with widespread HGPIN at the second biopsy cannot be fully determined by our data. However we feel that repeat biopsy should be advised after diagnosing widespread HGPIN on second biopsy, after adequately counseling patients on the risks and benefits of undergoing further prostate biopsies.

Moreover, we explored the prognostic value of other clinical and laboratory parameters on PCa. All parameters measured, including age, prostate volume, DRE, PSA, PSA ratio PSA density were not significant predictors of PCa on subsequent biopsy. Most studies have yielded similar results $(5,26-29)$, in that there does not appear to be any clinical parameter that helps identify men who are more likely to have cancer on further biopsies. Given these results, a finding of widespread HGPIN, especially on second biopsy, may be crucial in planning patients' future follow-up and should draw the urologist's attention, as it appears to be a significant predictor of PCa on further testing.

Of the neoplasms diagnosed on the third biopsy of our cohort, 9/15 (60\%) were low-grade, Gleason $6(3+3), 7$ of which showed a single core, $10-15 \%$ core involvement. Thus, $7 / 15$ (47\%) of the tumors identified are probably clinically insignificant and of 43 biopsies only 6 men had PCa with Gleason score $\geq 7$. It could be argued 
therefore that performing a third biopsy in men all with two diagnoses of HGPIN it may not be legitimate, as too many biopsies should to be performed to find one clinically significant cancer. However, if we restrict the analysis to patients with widespread lesions on second biopsy (17 men of 43), 9 tumors were identified, of which 4 were Gleason $\geq 7$. As such, 17 men underwent prostate biopsy to uncover 4 clinically significant high-grade cancers: these results in four men being biopsied to find one clinically significant cancer $(4: 1)$. These results suggests that, if not all men with two HGPIN biopsies should undergo further procedures, it may be appropriate to perform a repeat biopsy in men with widespread lesions on the second biopsy specimens, in order to uncover clinically significant prostate cancer.

It is correct to point out some limitations of this study as the small sample size $(n=43)$. Given the singularity of this group of patients, as it represents a second subset group of our initial study population, we believe that these results express the impact that widespread HGPIN on $\mathrm{PCa} .10$ patients who underwent prostate resection for bladder outlet obstruction were excluded from final analysis: given the different accuracy in PCa detection of TRUS-guided prostate biopsy vs. histologic analysis of resected specimen during transurethral prostatic surgery, we feel that such exclusion is justified (30). The follow-up period was limited to 12 months, time elapsed between the first and third biopsy: such period of time may seem inappropriate to evaluate the evolution of HGPIN on PCa, but patients are still under evaluation and the results of biopsies performed at 24 months will be soon available. Moreover, a significant number of patients failed to return for rebiopsy and unfortunately data on their follow-up was not available for analysis: however, if we consider these drop outs to be random, the results of this study should not have been significantly biased by such loss of data. This finding underlines the importance of patient follow-up after a diagnosis of HGPIN (3). Nevertheless, we must acknowledge that our study firstly confirmed in a homogeneous population that widespread HGPIN is associated with a significant higher risk of $\mathrm{PCa}$ even in patients with two previous biopsies. Furthermore another peculiar characteristics of our group is that our patients underwent three prostate biopsies in 12 months time regardless of PSA value, using the presence of HGPIN a mandatory indication for prostate biopsy. The lower cancer detection rate on initial biopsy and the high incidence of multiple isolated HGPIN areas may depend on our study population: our academic hospital operates under the Italian National Care System which does not support screening programs for $\mathrm{PCa}$. Furthermore, our clinical facility opened in 2002, and we can assume that our patient population had limited access to PCa centers and screening programs in the past.

\section{Conclusions}

The results of our study suggest that HGPIN and in particular widespread HGPIN are associated with an increased risk of PCA on a repeat biopsy in men with two previous diagnoses of HGPIN. No clinical parameter eval- uated such as age, PSA, prostate volume, DRE and PSA derivates was able to significantly predict $\mathrm{PCa}$ in this particular group of patients. Further studies are needed to confirm these findings in other populations and to evaluate which possible biological factors related to widespread HGPIN are responsible for the observed results.

\section{REFEREnCES}

1. Montironi R, Mazzucchelli R, Lopez-Beltran A, et al. Prostatic intraepithelial neoplasia: its morphological and molecular diagnosis and clinical significance BJU Int. 2011; 108:1394-401.

2. Epstein JI, Herawi M. Prostate needle biopsies containing prostatic intraepithelial neoplasia or atypical foci suspicious for carcinoma: implications for patient care J Urol. 2006; 175:820-34.

3. Maatman TJ, Papp SR, Carothers GGet al. The critical role of patient follow-up after receiving a diagnosis of prostatic intraepithelial neoplasia Prostate Cancer Prostatic Dis. 2001; 4:63-66.

4. Aboseif S, Shinohara K, Weidner N, et al. The significance of prostatic intra-epithelial neoplasia Br J Urol. 1995; 76:355-9.

5. Borboroglu PG, Sur RL, Roberts JL, Amling CL. Repeat biopsy strategy in patients with atypical small acinar proliferation or high grade prostatic intraepithelial neoplasia on initial prostate needle biopsy J Urol. 2001; 166:866-70.

6. Merrimen JL, Jones G, Srigley JR. Is high grade prostatic intraepithelial neoplasia still a risk factor for adenocarcinoma in the era of extended biopsy sampling? Pathology. 2010; 42:325-9.

7. Antonelli A, Tardanico R, Giovanessi L, et al. Predicting prostate cancer at rebiopsies in patients with high-grade prostatic intraepithelial neoplasia: a study on 546 patients Prostate Cancer Prostatic Dis. 2011; 14:173-6.

8. Chin AI, Dave DS, Rajfer J. Is repeat biopsy for isolated highgrade prostatic intraepithelial neoplasia necessary? Rev Urol. 2007; 9:124-31.

9. Godoy G, Taneja SS. Contemporary clinical management of isolated high-grade prostatic intraepithelial neoplasia Prostate Cancer Prostatic Dis. 2008; 11:20-31.

10. Srigley JR, Merrimen JL, Jones G, Jamal M. Multifocal highgrade prostatic intraepithelial neoplasia is still a significant risk factor for adenocarcinoma Can Urol Assoc J. 2010; 4:434.

11. Lee MC, Moussa AS, Yu C, et al. Multifocal high grade prostatic intraepithelial neoplasia is a risk factor for subsequent prostate cancer J Urol. 2010; 184:1958-62.

12. Bishara T, Ramnani DM, Epstein JI. High-grade prostatic intraepithelial neoplasia on needle biopsy: risk of cancer on repeat biopsy related to number of involved cores and morphologic pattern Am J Surg Pathol. 2004; 28:629-33.

13. Merrimen JL, Jones G, Walker D, et al. Multifocal high grade prostatic intraepithelial neoplasia is a significant risk factor for prostatic adenocarcinoma J Urol. 2009; 182:485-90; discussion 490.

14. Abdel-Khalek M, El-Baz M Ibrahiem el H. Predictors of prostate cancer on extended biopsy in patients with high-grade prostatic intraepithelial neoplasia: a multivariate analysis model BJU Int. 2004; 94:528-33.

15. Akhavan A, Keith JD, Bastacky SI, et al. The proportion of cores with high-grade prostatic intraepithelial neoplasia on extended-pattern needle biopsy is significantly associated with prostate cancer on site-directed repeat biopsy BJU Int. 2007; 99:765-9. 
16. De Nunzio C, Trucchi A, Miano R, et al. The number of cores positive for high grade prostatic intraepithelial neoplasia on initial biopsy is associated with prostate cancer on second biopsy J Urol. 2009; 181:1069-74; discussion 1074-5.

17. Raviv G, Janssen T, Zlotta AR, et al. Prostatic intraepithelial neoplasia: influence of clinical and pathological data on the detection of prostate cancer J Urol. 1996; 156:1050-4; discussion 1054-5.

18. De Nunzio C, Freedland SJ, Miano R, et al. Metabolic syndrome is associated with high grade gleason score when prostate cancer is diagnosed on biopsy. Prostate. 2011; doi: 10.1002/pros.21364. [Epub ahead of print].

19. Sakr WA D. M. A., Montironi R, Humphrey, et al. Prostatic Intraepithelial Neoplasia In: WHO Classification of Tumours: Pathology and Genetics of Tumours of the Urinary System and Male Genital Organs. Edited by JN Eble, G Sauter, JI Epstein and IA Sesterhenn. Lyon, France: IARC Press. 2004; 193:198.

20. Epstein JI HB, Algaba F, Humphrey PA, et al. Acinar Adenocarcinoma In: WHO Classification of Tumours: Pathology and Genetics of Tumours of the Urinary System and Male Genital Organs. Edited by JN Eble, G Sauter, JI Epstein and IA Sesterhenn. Lyon, France: IARC Press. 2004; 162-192.

21. Netto GJ, Epstein JI. Widespread high-grade prostatic intraepithelial neoplasia on prostatic needle biopsy: a significant likelihood of subsequently diagnosed adenocarcinoma Am J Surg Pathol. 2006; 30:1184-8

22. Goeman L, Joniau S, Ponette D, et al. Is low-grade prostatic intraepithelial neoplasia a risk factor for cancer? Prostate Cancer Prostatic Dis. 2003; 6:305-10.
23. Gokden N, Roehl KA, Catalona WJ, Humphrey PA. High-grade prostatic intraepithelial neoplasia in needle biopsy as risk factor for detection of adenocarcinoma: current level of risk in screening population Urology. 2005; 65:538-42.

24. Moore CK, Karikehalli S, Nazeer T, et al. Prognostic significance of high grade prostatic intraepithelial neoplasia and atypical small acinar proliferation in the contemporary era J Urol. 2005; 173:70-2.

25. Park S, Shinohara K, Grossfeld GD, Carroll PR. Prostate cancer detection in men with prior high grade prostatic intraepithelial neoplasia or atypical prostate biopsy J Urol. 2001; 165:1409-14.

26. Kamoi K, Troncoso P, Babaian RJ. Strategy for repeat biopsy in patients with high grade prostatic intraepithelial neoplasia J Urol. 2000; 163:819-23.

27. Postma R, Roobol M, Schroder FH, van der Kwast T. H. Lesions predictive for prostate cancer in a screened population: first and second screening round findings Prostate. 2004; 61:260-6.

28. Roscigno M, Scattoni V, Freschi M, et al. Monofocal and plurifocal high-grade prostatic intraepithelial neoplasia on extended prostate biopsies: factors predicting cancer detection on extended repeat biopsy Urology 2004; 63:1105-10.

29. Langer JE, Rovner ES, Coleman BG, et al. Strategy for repeat biopsy of patients with prostatic intraepithelial neoplasia detected by prostate needle biopsy J Urol. 1996; 155:228-31.

30. Jones JS, Follis HW, Johnson JR. Probability of finding T1a and $T 1 b$ (incidental) prostate cancer during TURP has decreased in the PSA era Prostate Cancer Prostatic Dis. 2009; 12:57-60.

\section{Correspondence}

Cosimo De Nunzio, MD, PhD (Corresponding Author)

cosimodenunzio@virgilio.it

Simone Albisinni, MD

albisinni.simone@gmail.com

Antonio Cicione, MD

acicione@libero.it

Costantino Leonardo, MD

costantino.leonardo@gmail.com

Francesco Esperto, MD

francescoesperto@gmail.com

Andrea Tubaro, MD

Department of Urology, Ospedale Sant'Andrea,

Università "La Sapienza"

Via di Grottarossa 1035 - 00198 Roma, Italy

Mauro Gacci, MD

Department of Urology, Ospedale Careggi, Università di Firenze

Largo Brambilla 3 - 50134 Firenze, Italy

maurogacci@yahoo.it 